\title{
Simple Microwave Technique for Non-Destructive Testing of Electrical Properties of Magnetized Materials
}

\author{
L. LAURINAVIČIUS \\ Department of Electrical Engineering, Vilnius Gediminas Technical University
}

Sauletekio av. 11, LT-10223 Vilnius, Lithuania

New technologies allow obtain low-dimensional structures, thin films, monocrystal samples of modern semiconductor materials. Electrical parameters of semiconductors should be tested by non-destructive simple methods to provide the high quality of new electronic devices. The principle of operation of proposed compact microwave meter is based on magnetic vortex oscillation and magnetoplasmic wave excitation technique in semiconductors placed in strong magnetic field. A high frequency field is interacting with charge carriers of semiconductor sample and contactless measurements of density $N$ and mobility $\mu$ of free charge carriers of semiconductors can be realized. Microwave technique for non-destructive testing electrical properties of semiconductor materials is described. Simple microwave meter consists of constant magnetic field source, high frequency generator, transmitting-receiving antenna and indicator. In semiconductor specimen placed in constant and alternating magnetic fields a vortex current and magnetoplasmic microwave are excited. The response signals are measured to find a value of density $N$ and mobility $\mu$ of free charge carriers in testing materials. Experimental parameters of measured $n$-InSb, CdHgTe, BiSb specimens are presented.

DOI: 10.12693 /APhysPolA.125.205

PACS: 72.80.Ey, 07.57.-c, 52.35.-g, 07.55.Db

\section{Introduction}

In microelectronic industry the goal is to drive costs down by bringing yields up at the same time. To reach this goal it is important to measure the parameters and analyze the defects existing in semiconductor materials and their impact on the latter device performance. The contactless non-destructive microwave technique for testing electrical properties of semiconductors is presented. The proposed methods do not require surface preparation of tested specimens.

Magnetoplasmic microwaves will propagate in solid-state plasma (metals or semiconductors) when a magnetic field is applied [1]. Main electrical properties of semiconductors are determined by values of density and mobility of free charge carriers. Microwave magnetoplasma methods are successfully used for investigations of semiconductors and semimetals due to their universality $[2,3]$. One of well known methods is the Rayleigh interferometry when transmitted via investigated specimen microwave signal is compared with reference signal. The reference signal has a constant phase and amplitude. The amplitude and wavelength of transmitted wave depend on electrical properties of a semiconductor. But as it is measurement equipment is complicated and there are problems to measure thin specimen, because transmitted signal is weak. The accuracy of measurement cannot be so high, because there are so many measured parameters: amplitudes of oscillation, magnetic induction and geometrical size of specimen.

The proposed methods to measure density and mobility of free charge carriers of semiconductors are based on magnetoplasma phenomenon to absorb the HF signal. $\mathrm{HF}$ resonator of lumped elements is proposed.

\section{Theoretical background of investigation}

Consider a situation where magnetic field polarized in the $x$ direction, with a time dependence $H_{x}=H \cos \omega t$ and propagating in the $z$ direction into the body of a conductor (semiconductor) as in Fig. 1a. Let the conductor infinity large surface be parallel to the $x y$ plane and the $z$ axis be directed perpendicular to it. For the moment we set static magnetic field $H_{0}=0$.

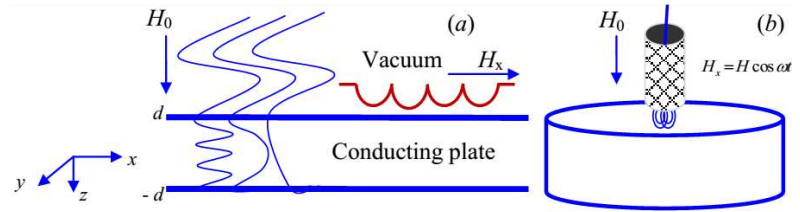

Fig. 1. Transverse linearly polarized electromagnetic wave incident normally on a surface of infinitely large conductor, placed into the strong constant magnetic field $H_{z}=H_{0}$ (a). Conducting plate with an electric coil placed on its surface (b).

The electromagnetic waves are damped exponentially inside the conductor, with a penetration length given by the skin depth:

$$
\delta=\left(\frac{2}{\omega \mu_{0} \sigma}\right)^{1 / 2},
$$

where $\sigma$ - conductivity of the conductor.

At frequencies higher than the plasma frequency $\omega_{\mathrm{p}}^{2}=$ $\frac{4 \pi N e^{2}}{m}$, where $N, e, m$, are the density, charge, and effective mass of electrons, the displacement current dominates over the conduction current and a conductor becomes transparent.

If a large static magnetic field $H_{z}=H_{0}$ is applied, the behaviour is quite different because of the alternating Hall electric field and microwaves can propagate in conductor along the direction of magnetic induction. These waves will propagate with little damping if the electron cyclotron frequency $\omega_{\mathrm{c}}=\frac{e H}{m c}$ is much greater than the 
average frequency of electron scattering $\omega_{\mathrm{c}} \gg \frac{1}{\tau}$, where the relaxation time $\tau$ is the mean time between electron collisions.

Propagating waves in a conducting medium in a static magnetic field were first observed in the ionosphere and were called "whistlers" or "helicons". The static field for whistlers is the Earth magnetic field $(\approx 0.5 \mathrm{G})$, which is much smaller than the field required in metal or semiconductor.

Inside the conductor, the electric field $\bar{E}$, magnetic field $\bar{H}$, and the current density $\bar{J}$ have the same time dependence $\mathrm{e}^{\mathrm{i} \omega t}$ as the incident wave.

The conductivity $\sigma$ of the plate is provided by the electrons with an isotropic mass. In the presence of a magnetic field, in addition to the electric field, the charge experiences an additional field due to the Lorentz force. Ohm's law $\bar{J}=\sigma \bar{E}$ has an additional term and we have the following equation of motion of carriers:

$$
m \frac{\mathrm{d} \bar{V}}{\mathrm{~d} t}=e \bar{E}+e(\bar{V} \times \bar{B})-m\left(\frac{\bar{V}}{\tau}\right),
$$

where $\bar{V}$ is drift velocity.

The equations of motion (2) must be solved with the Maxwell equations

$$
\operatorname{rot} \bar{E}=-\mu_{0} \frac{\partial \bar{H}}{\partial t}, \quad \operatorname{rot} \bar{H}=\varepsilon_{L} \varepsilon_{0} \frac{\partial \bar{E}}{\partial t}+\bar{J} .
$$

Taking into account that $\omega \ll \tau^{-1}=10^{12} \mathrm{~Hz}$ and ignoring the displacement currents in comparison with the conductive ones we obtain a system of equations for the complex amplitudes $H_{x}$ and $H_{y}[4]$ :

$$
\begin{aligned}
& c^{2} \frac{\partial^{2}}{\partial z^{2}} H_{x}-\mathrm{i} \omega \sigma_{11} H_{x}-\mathrm{i} \omega \sigma_{12} H_{y}=0, \\
& c^{2} \frac{\partial^{2}}{\partial z^{2}} H_{y}-\mathrm{i} \omega \sigma_{22} H_{y}+\mathrm{i} \omega \sigma_{12} H_{x}=0,
\end{aligned}
$$

where the components of the conductivity tensor $\sigma$ are

$$
\begin{aligned}
\sigma_{11} & =\sigma_{22}=\sigma_{0} \frac{1}{1+\left(\frac{e H \tau}{m c}\right)^{2}}, \\
\sigma_{12} & =\sigma_{0} \frac{\frac{e H \tau}{m c}}{1+\left(\frac{e H \tau}{m c}\right)^{2}}, \quad \sigma_{0}=\frac{N e^{2} \tau}{m} .
\end{aligned}
$$

The boundary conditions have the following form (see Figure 1):

$$
\begin{aligned}
& H_{x}=H, \quad H_{y}=0 \quad \text { if } \quad z= \pm d, \\
& \frac{\partial H_{x}}{\partial z}=\frac{\partial H_{y}}{\partial z}=0 \quad \text { if } \quad z=0 .
\end{aligned}
$$

The solutions of Eqs. (4), satisfying (6), are

$$
\begin{aligned}
& H_{x}=\frac{1}{2} H\left(\frac{\cos k_{-} z}{\cos k_{-} d}+\frac{\cos k_{+} z}{\cos k_{+} d}\right), \\
& H_{y}=\frac{1}{2} \mathrm{i} H\left(\frac{\cos k_{+} z}{\cos k_{+} d}-\frac{\cos k_{-} z}{\cos k_{-} d}\right) .
\end{aligned}
$$

The propagation of magnetoplasma wave $\left(k_{ \pm}\right)$is determined by dispersion equation

$$
c^{2} \frac{k_{ \pm}^{2}}{\omega^{2}}=\varepsilon_{L}\left(1-\frac{\omega_{\mathrm{p}}^{2}}{\omega\left[\left(\omega \pm \omega_{\mathrm{c}}\right)+\mathrm{i} \nu\right]}\right) .
$$

If the incident wave is linearly polarized, both circularly polarized waves $\left(k_{+}, k_{-}\right)$will be excited.

To have a propagating wave we must have $|\operatorname{Re}(k)| \gg$ $|\operatorname{Im}(k)|$ which means that $k^{2}$ must be positive and largely real. For $k^{2}$ to be largely real, $\omega_{\mathrm{c}} \tau$ must be large compared to 1 . For free electron conductor $(-)$ wave propagates and $(+)$ wave is attenuated.

If $\left(\omega \pm \omega_{\mathrm{c}}\right) \gg \nu ; \omega_{\mathrm{c}} \gg \omega ; \omega_{\mathrm{c}} \tau \gg 1$, real part of complex relative dielectric constant for helicon wave looks as follows:

$$
\varepsilon^{\prime}=\omega_{\mathrm{p}}^{2} /\left(\omega \omega_{\mathrm{c}}\right),
$$

and half wavelength of helicons can be calculated $\left(k^{\prime}=\right.$ $\left.(\omega / c) \sqrt{\varepsilon^{\prime}}\right)$ :

$$
\frac{\lambda}{2}=4 \pi c /\left(\omega \sqrt{\varepsilon^{\prime}}\right) \text {. }
$$

The magnetic flows are

$$
\begin{aligned}
& \Phi_{x}=\int_{-d}^{d} H_{x} \mathrm{~d} z=H\left(\frac{\tan k_{-} d}{k_{-}}+\frac{\tan k_{+} d}{k_{+}}\right), \\
& \Phi_{y}=\int_{-d}^{d} H_{y} \mathrm{~d} z=\mathrm{i} H\left(\frac{\tan k_{+} d}{k_{+}}-\frac{\tan k_{-} d}{k_{-}}\right) .
\end{aligned}
$$

From (11) we can determine magnetic permeability $\mu_{L}$ :

$$
\mu_{L}=\frac{\Phi_{x}}{2 d H}=\frac{1}{2 d}\left(\frac{\tan k_{-} d}{k_{-}}+\frac{\tan k_{+} d}{k_{+}}\right) .
$$

The impedance $Z$ of the inductive coil is purely active

$$
Z=\mathrm{i} \omega L_{0} \mu_{L}=\frac{2 \omega_{\mathrm{c}} \tau}{n^{2} \pi^{2}} \omega L_{0},
$$

where $L_{0}$ is the coil's inductivity without the semiconductor, $n$ is $1,2,3 \ldots$

\section{Experimental approach}

The principle of operation of proposed compact microwave meter is based on magnetic vortex oscillation and magnetoplasmic wave excitation in semiconductors placed in strong magnetic field. The experimental arrangement of compact microwave technique for exciting and detecting of channeled electromagnetic waves and magnetic vortex oscillations in solid-state plasma is shown in Fig. 2.

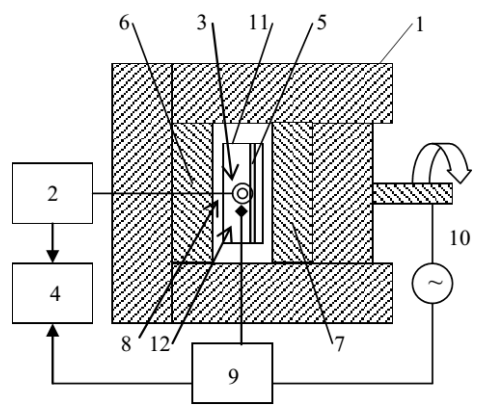

Fig. 2. Block diagram of magnetoplasma meter. 


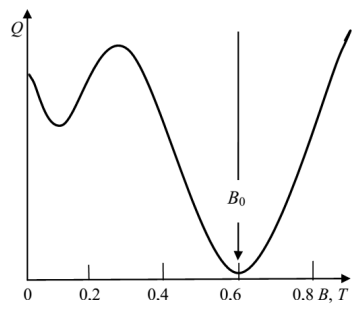

Fig. 3. Measurement of density. High frequency signal dependence from magnetic induction $B$.

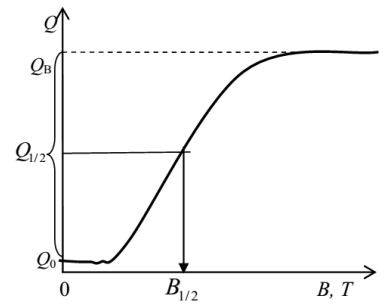

Fig. 4. Measurement of mobility. High frequency signal dependence from magnetic induction $B$.

Simple microwave meter consists of constant magnetic field source 1 , high frequency generator 2, transmitting-receiving antenna 3 and indicator 4 . A semiconductor specimen 5 is put in strong magnetic field system with permanent magnets $(6,7)$ made of rare-earth metals. The intensity of constant magnetic field in the gap 8 is regulated by control system 9 and actuator 10 . The magnetoplasma wave is excited in local area of a semiconductor plate put in a constant magnetic field. Propagating across semiconductor plate magnetoplasma wave is received by the same coil 3. A receiving signal is indicated by memorized oscilloscope 4 . Magnetic field control sys- tem 9 is connected to the oscilloscope 4 and motor of actuator 10. Described microwave meter has also a cryogenic system 11 and magnetic field sensor 12 .

Set of two magnet $50.8 \mathrm{~mm}$ long $\times 50.8 \mathrm{~mm}$ wide $\times$ $25.4 \mathrm{~mm}$ thick is used for magnetic system. Material: neodymium $(\mathrm{NdFeB})$ or samarium cobalt $(\mathrm{SmCo})$. The maximum energy products of these rare-earth alloys range from $20 \mathrm{MGOe}$ to $32 \mathrm{MGOe}$, which is approximately $160-260 \mathrm{~kJ} / \mathrm{m}^{3}$.

Quality $Q$ of induction coil (LC-resonator) placed on the surface of semiconductor in magnetic fields, when frequency $\omega$ of high frequency generator is $\omega \approx 2 \pi f_{\mathrm{R}}$ is very low, because $Q=\frac{\sqrt{L / C}}{R}$ and resistance losses $R$ is high (mine dimensional resonance of helicon waves). When mine (half-length) resonance is observed $\omega=\omega_{R}$, the value of density $N$ is determined from Fig. 3 by simple equation

$$
N=\frac{\varepsilon_{0} c^{2} B_{0}}{2 e d^{2} f_{\mathrm{R}}} .
$$

When exciting frequencies $\omega \ll 2 \pi f_{\mathrm{R}}$, we have non-resonance conditions in the specimen. In this case values of density $N$ and mobility $\mu$ are possible to be determined by analysing tensor of specimen conductivity $|\sigma|$.

Quality of LC-resonator $Q$ is described by equation

$$
Q \equiv \sigma_{x x}=\frac{\sigma_{z z}}{1+\mu^{2} B^{2}}=\frac{e N \mu}{1+\mu^{2} B^{2}},
$$

where $e, N, \mu$ are charge, density and mobility of electrons, respectively.

Mobility of electrons can be calculated (Fig. 4) very simply

$$
\mu=\frac{1}{B_{1 / 2}},
$$

and quality $Q_{1 / 2}=\left(Q_{\mathrm{B}}-Q_{0}\right) / 2$.

Measured values of density and mobility of free charge carriers in InSb, CdHgTe, and BiSb.

TABLE

\begin{tabular}{c|c|c|c|c}
\hline \hline Investigated material & TS density $\left[\mathrm{m}^{-3}\right]$ & Mobility $\left[\mathrm{m}^{2} \mathrm{~V}^{-1} \mathrm{~s}^{-1}\right]$ & Measured values density $\left[\mathrm{m}^{-3}\right]$ & ${\text { Mobility }\left[\mathrm{m}^{2} \mathrm{~V}^{-1} \mathrm{~s}^{-1}\right]}$ \\
\hline $\mathrm{InSb}$ & $2.4 \times 10^{22}$ & 6.8 & $2.3 \times 10^{22}$ & 6.9 \\
$\mathrm{InSb}$ & $1.4 \times 10^{20}$ & 68 & $1.4 \times 10^{20}$ & 62 \\
$n-\mathrm{InSb}$ & $0.8 \times 10^{23}$ & 4.8 & $0.85 \times 10^{23}$ & 5.0 \\
$\mathrm{CdHgTe}$ & $3.0 \times 10^{23}$ & 3.6 & $2.8 \times 10^{23}$ & 3.8 \\
$\mathrm{BiSb}$ & $1.8 \times 10^{23}$ & 48 & $1.65 \times 10^{23}$ & 45
\end{tabular}

\section{Conclusion}

Measurements of density and mobility of free charge carriers were executed in monocrystallic specimens of $\mathrm{InSb}, \mathrm{CdHgTe}, \mathrm{BiSb}$.

We used static magnetic field with induction $B<$ 1.2 T. Good coincidence with technical specification (TS) of specimens was confirmed. Semiconductor samples were investigated at room and liquid nitrogen temperatures. Some experimental results are put in Table.

Proposed contactless non-destructive microwave technique for measurement of electrical properties of semi- conductors can be used in express testing of thin films of magnetized materials.

\section{References}

[1] B.W. Maxfield, Am. J. Phys. 37, 241 (1969).

[2] J. Pozhela, Plasma and Current Instabilities in Semiconductors, Pergamon Press, Oxford 1981.

[3] L. Laurinavičius, The Use of HF Waves in NonContact Testing of Narrow-Gap Semiconductors and High-T $T_{C}$ Superconductors, Technika, Vilnius 1998.

[4] Z. Jankauskas, L. Laurinavicius, Electron. Electr. Eng. 37, 32 (2002). 